\title{
ESTUDOS DA BIODIVERSIDADE DE AMEBAS TESTÁCEAS PARA ESTRATÉGIAS VOLTADAS À PRESERVAÇÃO: UMA REVISÃO
}

\author{
Rodrigo Leite Arrieira* \\ Leilane Talita Fatoreto Schwind** \\ Geziele Mucio Alves**** \\ Fábio Amodêo Lansac-Tôha**
}

RESUMO: As planícies de inundação são ecossistemas que possuem uma ampla variedade de ambientes aquáticos, sendo caracterizados por sua grande complexidade funcional, estrutural e elevada diversidade de organismos. Dentre os organismos estudados em ambientes de planície de inundação, destacam-se as amebas testáceas que são um grupo polifilético de protozoários de vida livre. As amebas testáceas apresentam diversas vantagens em estudos ambientais, devido a uma série de características interessantes em investigações ecológicas, tais como elevada tolerância a alterações das condições ambientais e seu ciclo de vida curto, que permitem investigações sobre processos demográficos tanto em escala espacial quanto temporal, além de responder sobre o funcionamento de todo ecossistema. Diante disso, esse trabalho teve como objetivo realizar uma revisão sobre a biodiversidade das amebas testáceas em planícies de inundação, com enfoque na contribuição do grupo para estratégias ligadas à proteção ambiental e delineamento de áreas prioritárias à conservação.

PALAVRAS-CHAVE: Conservação ambiental; Ecologia de comunidades; Plâncton.

\section{A REVIEW OF STUDIES ON THE BIODIVERSITY OF TESTATE AMOEBAE FOR PRESERVATION STRATEGIES}

ABSTRACT: Floodplains are ecosystems with a great variety of water environments. They are characterized by great functional and structural complexity and high

Doutorado em Biologia das Interações Orgânicas pela Universidade Estadual de Maringá (UEM), Brasil; E-mail: rodrigoarrieira@yahoo.com.br

** Doutorado em Ecologia de Ambientes Aquáticos Continentais pela Universidade Estadual de Maringá (UEM), Brasil

*** Doutorado e pós-doutorado em Ecologia de Ambientes Aquáticos Continentais pela Universidade Estadual de Maringá (UEM), Brasil

**** Doutorado em Oceanografia Biológica Ambientes Aquáticos Continentais pela Universidade de São Paulo; Docente titular do Departamento de Biologia da Universidade Estadual de Maringá (UEM), Brasil. Pesquisador na área de Ecologia, pelo Núcleo de Pesquisas em Limnologia, Ictiologia e Aquicultura (Nupélia) na Universidade Estadual de Maringá (UEM), Brasil. 
diversity of organisms. Testate amoebae, a polyphyletic group of free protozoa, are underscored among organisms in floodplains. Testate amoebae have several advantages within environmental studies due to a series of interesting characteristics in ecological investigations, such as high tolerance to changes in environmental conditions and short life cycle, which permit investigations on demographic processes at space and temporal scales. They are also sensitive within the functioning of the entire ecosystem. Current research reviews the biodiversity of testate amoebae in floodplains with special reference to the contribution of the group for strategies linked to environmental protection and design of conservational priority areas.

KEY WORDS: Environmental conservation; Ecology of communities; Plankton.

\section{INTRODUÇÃO}

Em regiões tropicais, as condições geológicas, hidrológicas e climáticas são favoráveis para a ocorrência de áreas alagáveis, tais como as planícies de inundação. Cerca de 5\% do Brasil são ocupados por estas áreas que são formadas por sistemas aquáticos rasos, dinâmicos e complexos, e ocorrem, geralmente, em terrenos de baixa declividade, com presença de distintos ambientes associados ao rio principal (GOPAL, 1994; ESTEVES, 2011).

As planícies de inundação formam macrossistemas fluviais caracterizados pela heterogeneidade de hábitats (JUNK; BALEY; SPARKS, 1989; TOCKNER; MALARD; WARD, 2000), com grande complexidade funcional e estrutural (NEIFF, 2001; WARD et al., 2002), além de elevada diversidade de espécies (TUNDISI; MATSUMURATUNDISI, 2008), sendo considerados ecossistemas com elevada produtividade (THOMAZ et al., 2003). A principal característica desses sistemas é a sazonalidade marcada pelo nível hidrológico que determinam seus pulsos de energia e matéria, sendo considerada a principal função de força sobre o funcionamento desses ecossistemas (JUNK, 1996; THOMAZ; ROBERTO; BINI, 1997).

Essas flutuações do volume de água, caracterizadas por dois períodos (cheia e seca), além de ser uma das principais responsáveis pelas alterações abióticas nos ambientes, também conduzem a organização das comunidades aquáticas nesses ecossistemas (JUNK; BALEY; SPARKS, 1989; NEIFF, 1990). Durante o período de 
cheia ou águas altas, os sistemas aquáticos expandem suas áreas, inundando porções da planície, antes isoladas, e estabelecendo ligações entre os diferentes tipos de ambientes. Essa conectividade pode ocorrer entre os ambientes e entre os próprios compartimentos dentro de cada ambiente, promovendo a troca de organismos (NEIFF, 1996). Em contraste, no período de seca ou águas baixas, os ambientes ficam mais rasos, ocorre o aumento da entrada de matéria orgânica e o incremento de nutrientes na coluna de água (CARVALHO et al., 2001; ROBERTO et al., 2009). Com isso, pode ser observado um aumento da produtividade primária dos organismos planctônicos (BONECKER et al., 2013), tais como as amebas testáceas.

Tais alterações, aliadas à heterogeneidade de ambientes e as interações dentro e entre as comunidades aquáticas, propiciam o desenvolvimento de uma elevada biodiversidade de organismos em planícies de inundação (ROCHA; THOMAZ, 2004; LANSAC-TÔHA et al., 2009). Dentre os trabalhos realizados em ambientes de planície de inundação, destacam-se aqueles realizados com as amebas testáceas (VELHO; BINI; LANSAC-TÔHA, 2004; ALVES et al., 2007; 2008; LANSACTÔHA et al., 2007).

As amebas testáceas são um grupo polifilético de protozoários de vida livre, em que uma única célula está inserida em uma carapaça com uma abertura através da qual pseudópodes sobressaem durante a locomoção ou alimentação (SMITH; BOBROV; LARA, 2008). São heterótrofos, podendo se alimentar de matéria orgânica, algas, células de plantas, protistas, fungos e pequenos metazoários. Algumas espécies são polífagas (alimentando-se de diversos tipos de alimentos), enquanto outras possuem alimentos específicos (GILBERT et al., 2000).

As pesquisas realizadas com amebas testáceas podem representar diversas vantagens em investigações ecológicas, devido a uma série de características interessantes que permitem responder sobre o funcionamento de todo ecossistema (LANSAC-TÔHA; VELHO; BONECKER, 2004; SCHWIND et al., 2013). Dentre essas vantagens, destacam-se sua elevada tolerância a alterações das condições ambientais e seu ciclo de vida curto, que permitem investigações sobre processos demográficos tanto em escala espacial quanto temporal (PENNAK, 1953; HARDOIM, 1997; DABÉS; VELHO, 2001). Além disso, esses organismos são considerados como bioindicadores da qualidade da água (JASSEY et al., 2011; SONG et al., 2014) e podem ser usados, 
também, em estudos de paleontologia devido à preservação de suas carapaças por um longo tempo nos ambientes (CHARMAN, 2001).

Dessa forma, as investigações ecológicas, que buscam ampliar o entendimento sobre a comunidade de amebas testáceas, são de grande importância para a manutenção da biodiversidade dos ambientes aquáticos, bem como para a implementação de medidas que visem a preservação dos ecossistemas aquáticos (RODRIGUES; BICUDO; MOSCHINI-CARLOS, 2003). Diante disso, esse trabalho teve como objetivo realizar uma revisão sobre a biodiversidade das amebas testáceas em planícies de inundação brasileiras, com enfoque na contribuição do grupo para estratégias ligadas à proteção ambiental e delineamento de áreas prioritárias à conservação em ambientes aquáticos.

\section{CARACTERIZAÇÃO DOS AMBIENTES DE PLANÍCIES DE INUNDAÇÃO}

Em planícies de inundação, além das trocas diretamente provocadas pela dinâmica das flutuações dos níveis de água, as inundações resultam em pulsos de nutrientes a partir da decomposição da vegetação da várzea (THOMAZ; ROBERTO; BINI, 1997). Atualmente, os ciclos biogeoquímicos são afetados pelos reservatórios a montante, por meio de dois processos: alteração dos padrões naturais do pulso de inundação, que se reflete nas trocas de água e, em certos anos, na ausência de inundações; e retenção de nutrientes nos reservatórios, especialmente fósforo (AGOSTINHO; VAZOLLER; THOMAZ, 1995; MANTOVANO et al., 2015).

Os estudos realizados em planícies de inundação destacam a importância desses ambientes, devida sua elevada biodiversidade de espécies (TUNDISI; MATSUMURA-TUNDISI, 2008). No Brasil, as planícies de inundação com maior representatividade de espécies são: Amazônica, Araguaia, Pantanal Mato-grossense e Paraná (Figura 1). 


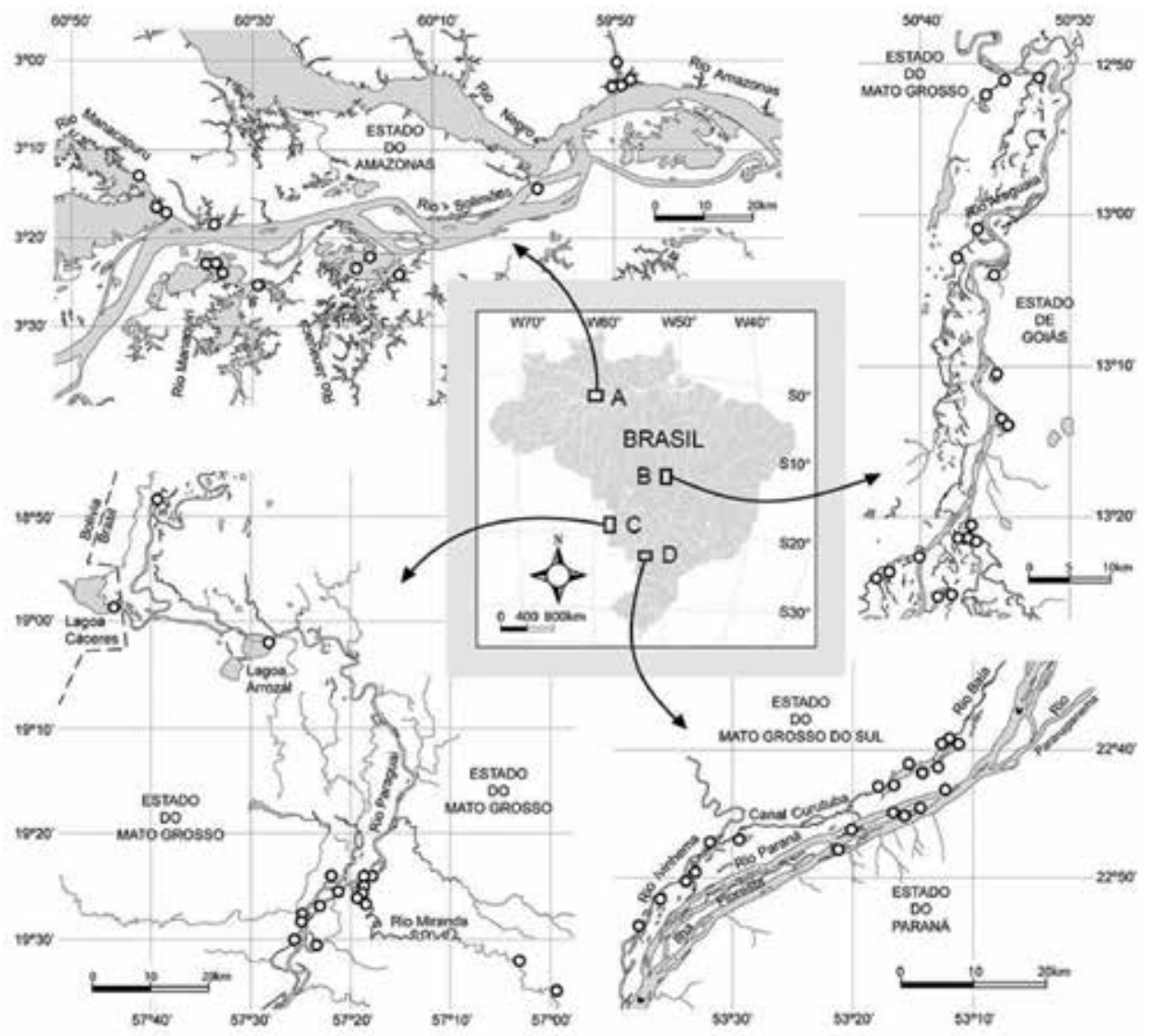

Figura 1. Planícies de inundação brasileiras com maiores representatividades de espécies: A) Amazônica; B) Araguaia; C) Pantanal Mato-grossense; C) Paraná.

A planície de inundação amazônica é constituída por uma complexa rede de lagoas e abrange uma área de $300.000 \mathrm{~km}^{2}$ (JUNK; BALEY; SPARKS, 1989). É considerada a maior área alagável brasileira e classificada de acordo com as propriedades físicas e químicas da água em dois principais tipos: várzea e igapó. Planícies de várzea são periodicamente inundadas por rios de água branca, como o Amazonas e seus tributários Purus e Juruá, que atingem cerca de 200.000 km², possuem $\mathrm{pH}$ próximos do neutro e elevada produtividade. Em contraste, planícies de igapó são áreas geologicamente antigas e erodidas da Guiana e região Central do Brasil, inundadas por águas pretas e caracterizadas pela baixa fertilidade (JUNK, 
1993). A amplitude da flutuação do nível das águas varia entre os rios da planície de inundação de ano para ano, e depende da precipitação (FERREIRA et al., 2010).

A bacia do rio Araguaia possui uma área total de aproximadamente 377.000 $\mathrm{km}^{2}$ com uma vazão média de $6.420 \mathrm{~m}^{3} / \mathrm{s}$ e a precipitação varia entre $1.300 \mathrm{~mm} /$ ano na alta bacia (Alto Araguaia) e mais de $2.000 \mathrm{~mm} /$ ano próximo à confluência com o rio Tocantins. O rio Araguaia é considerado um rio de baixa sinuosidade e seus principais afluentes são os rios do Peixe, Claro, Vermelho, Crixás-açu, Formoso e Caiapó na margem direita, e os rios Diamantino, Cristalino e das Mortes na margem esquerda. O rio das Mortes, que apresenta um padrão sinuoso e localizado paralelamente ao rio Araguaia, é seu mais importante tributário, compreendendo cerca de $60.000 \mathrm{~km}^{2}$ de área de drenagem (LATRUBESSE; STEVAUX, 2002).

A planície de inundação do Pantanal Mato-grossense é uma das maiores áreas úmidas contínuas do planeta, podendo ser delimitada em dez sub-regiões, com características geomorfológicas e ecológicas próprias, com diferentes níveis e duração das inundações. Apresenta uma elevada produtividade biológica que se reflete em grande abundância de organismos. A planície de inundação possui uma grande heterogeneidade e multiplicidade de tipos de corpos de água, com rios meândricos e anastomosados (ou seja, rios com curvas sinuosas e redes de canais que se bifurcam), lagoas e pequenos cursos de água intermitentes de aporte e drenagem, denominados "corixos" e "corixões" (com leito definido) e "vazantes" (CARVALHO, 1986).

Dentre as planícies de inundação brasileiras, pode se destacar a planície de inundação do alto rio Paraná. Esse sistema está localizado à margem oeste do rio Paraná entre os Estados de Mato Grosso e Paraná (SOUZA-FILHO; STEVAUX, 2004). O rio Paraná é formado pela junção dos rios Grande e Paranaíba, sendo considerado o principal rio da bacia da Prata, décimo maior em descarga do mundo $\left(5 \times 108 \mathrm{~m}^{3}\right.$ / ano) e quarto maior em área de drenagem $\left(2,8 \times 106 \mathrm{~km}^{2}\right.$ ) (AGOSTINHO; THOMAZ; NAKATANI, 2001). Este rio drena toda região Centro-Sul da América Central, dos Andes até a Serra do Mar, próximo ao Oceano Atlântico. O rio Paraná é afetado no seu curso por atividades antropogênicas, como descarga de agrotóxicos, nutrientes da agricultura e esgoto doméstico, remoção da vegetação ripária e, principalmente, a construção de reservatórios (AGOSTINHO; THOMAZ; GOMES, 2004). 
A planície de inundação do alto rio Paraná está reduzida a um pequeno trecho $(230 \mathrm{~km})$ entre os reservatórios de Porto Primavera e Itaipu. Este trecho caracteriza o último sistema rio-planície de inundação não represado desse rio em território brasileiro. Devido à sua grande importância, três unidades de conservação foram criadas: Área de Proteção Ambiental das Ilhas e Várzeas do rio Paraná, Parque Nacional de Ilha Grande e Parque Estadual do Ivinhema (THOMAZ; AGOSTINHO; HAHN, 2004).

Tendo em vista sua elevada variabilidade de hábitats terrestres e aquáticos, a planície de inundação do alto rio Paraná ainda possui uma grande diversidade de espécies, em que o pulso de inundação é a principal força reguladora da estruturação e funcionamento das comunidades (JUNK; BALEY; SPARKS, 1989). Embora alterada pelos reservatórios localizados à montante, os períodos hidrológicos seguem certa sazonalidade, contribuindo para a manutenção de muitas espécies adaptadas a estas condições (AGOSTINHO; THOMAZ; GOMES, 2004).

Este sistema apresenta vários tipos de ambientes aquáticos e de transição terra-água, que se distinguem pela sua morfometria, hidrodinâmica e pelo grau de conexão com o rio principal e tributários secundários (AGOSTINHO; ZALEWSKI, 1996). Tais diferenças conferem a esses ambientes características limnológicas próprias que controlam os padrões de distribuição dos organismos e a dinâmica funcional dos sistemas (THOMAZ; ROBERTO; BINI, 1997). Este grande número de ambientes aquáticos, tais como rios, lagoas abertas e fechadas, ressacos e canais, são categorizados como lóticos, semilóticos e lênticos. Esses tipos de ambientes são caracterizados por serem formados a partir do grau de conectividade com o rio principal e as complexas interações entre a superfície da água e o lençol freático (STANFORD; WARD, 1988; WARD; STANFORD, 1995).

\section{ESTUDOS COM AMEBAS TESTÁCEAS}

As amebas testáceas são organismos considerados essencialmente aquáticos, possuem ampla distribuição e podem ser registradas em diversos hábitats: macrófitas (DABÉS; VELHO, 2001; ARRIEIRA et al., 2015a), sedimento (ALVES et al., 2010; 2012), 
turfeiras (BOBROV; CHARMAN; WARNER, 1999; MITCHELL; CHARMAN; WARNER, 2008) e solos (BEYENS; MEISTERFELD, 2001; FERNÁNDEZ, 2015). Além disso, estudos registraram elevadas densidades desses organismos no plâncton (LANSACTÔHA et al., 2000; VELHO et al., 2000; ARRIEIRA et al., 2015b; SCHWIND et al., 2016).

A importância das amebas testáceas associadas às macrófitas aquáticas tem sido enfatizada em diversos trabalhos (TORRES; JEBRAN, 1994; TORRES; SCHWARZBOLD, 2000; DABÉS; VELHO, 2001; LANSAC-TÔHA; VELHO; BONECKER, 2003; ALVES et al., 2012). A elevada ocorrência e a abundância desses organismos em áreas colonizadas por macrófitas aquáticas pode ser atribuída à elevada produtividade e heterogeneidade proporcionada por essas plantas (HARDOIM; HECKMAN, 1996; SOUZA, 2005). Essa afirmação foi corroborada por Hardoim (1997), que constatou que as amebas testáceas se beneficiam dos microhábitats proporcionados pelas macrófitas, pois os processos de decomposição dos detritos nesses locais são intensos e permitem a proliferação de fungos, algas perifíticas, bactérias e ciliados, que podem servir de alimento para as amebas. Além disso, a autora afirmou que as macrófitas fornecem abrigo contra a predação de invertebrados e, consequentemente, favorecem o estabelecimento das espécies nesse hábitat.

Os hábitats de turfeiras são filmes de água encontrados em regiões de solo alagado com húmus orgânico e alta cobertura de musgo (Sphagmum) (FOURNIER et al., 2012). Apresentam umidade, luz, temperatura, concentrações de oxigênio e pH adequados para a ocorrência de amebas testáceas (MITCHELL et al., 1999).

As amebas testáceas constituem, também, uma parte considerável da biota de solos úmidos, em termos de biomassa e diversidade de espécies (FOISSNER, 1987). Respondem de forma rápida às mudanças de umidade, concentrações de matéria orgânica, nutrientes e composição e tamanhos das partículas do solo (TSYGANOV; EMBULAEVA; MAZEI, 2012).

Em ambientes aquáticos, as alterações na estrutura e dinâmica das comunidades de amebas testáceas são fenômenos muito relevantes, não somente para a própria comunidade, mas também para o funcionamento de todo o ecossistema (LANSAC-TÔHA et al., 2009). Isto porque as amebas testáceas podem apresentar um importante papel na organização das comunidades aquáticas, pois representam um 
elo essencial na transferência de matéria e energia entre produtores e consumidores na cadeia alimentar em sistemas aquáticos (LANSAC-TÔHA et al., 2004).

A densidade e riqueza de amebas testáceas estão entre as maiores observadas no plâncton de ambientes de planície de inundação. Esse fato deve-se principalmente à habilidade desses organismos em colonizar vários ambientes que caracterizam este ecossistema, devido às suas estratégias alimentares e reprodutivas bem sucedidas (LANSAC-TÔHA; VELHO; BONECKER, 2004). Na Figura 2 podem ser observadas algumas espécies que ocorrem em ambientes de planícies de inundação.

A

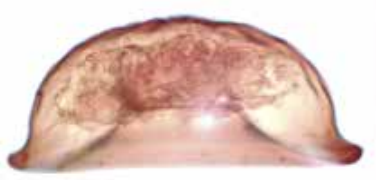

$30 \mu \mathrm{m}$
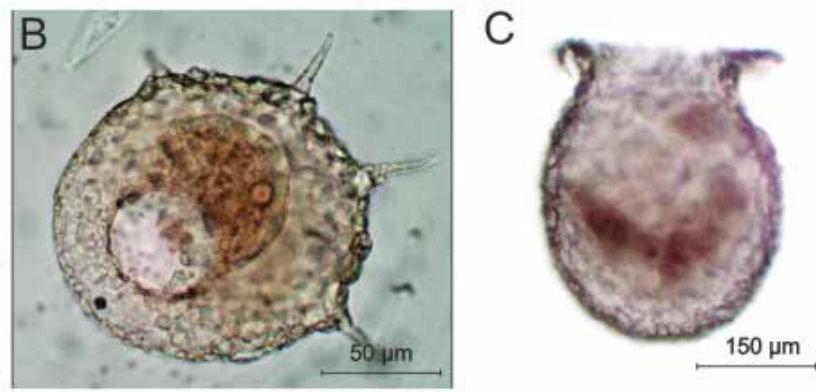

Figura 2. Espécies de amebas testáceas de ambientes de planícies de inundação:

A) Arcella vulgaris; B) Centropyxis aculeata; C) Difflugia urceolata

Fonte: Elaborado pelos autores

As amebas testáceas têm sido estudadas em diferentes biótopos aquáticos, tais como sedimento, macrófitas aquáticas, plâncton de rios, reservatórios e lagos (BALIK; SONG, 2000; SOUZA, 2005; LANSAC-TÔHA et al., 2008; TODOROV; GOLEMANSKY; BLAGOVEST, 2008; SNEGOVAYA; ALEKPEROV, 2009). Em um levantamento realizado em ambientes aquáticos continentais brasileiros foram reportadas 282 espécies no plâncton, 131 espécies associadas às macrófitas aquáticas na região litorânea e 72 espécies no sedimento (LANSAC-TÔHA et al., 2007). O maior número de espécies registradas no plâncton pode ser explicado em virtude da maioria dos estudos realizados no Brasil terem sido realizados no compartimento planctônico (VELHO; LANSAC-TÔHA; BINI, 1999; LANSAC-TÔHA et al., 2007).

Em relação à biodiversidade desses organismos, em um estudo conduzido na região planctônica de diferentes tipos de ambientes (incluindo lagoas conectadas 
e isoladas, ressacos, rios e canais), na planície de inundação do alto rio Paraná, foram identificadas 120 espécies de amebas testáceas (LANSAC-TÔHA et al., 2009). Nessa mesma planície, em outro trabalho avaliando a diversidade de espécies em diferentes hábitats de uma lagoa, foram registradas 53 espécies no plâncton, 71 espécies nas macrófitas e 82 espécies no sedimento (ALVES et al., 2010). Esse estudo constatou, ainda, que a presença desses organismos no plâncton não deve ser atribuída a processos estocásticos, uma vez que a diversidade de espécies foi notavelmente elevada em relação à diversidade total do ambiente. Algumas espécies planctônicas possuem vacúolos de gás em seu interior que permitem que permaneçam na coluna de água por maior tempo (VELHO; LANSAC-TÔHA; BINI, 2003).

\section{PERSPECTIVAS AMBIENTAIS PARA ESTUDOS COM AMEBAS TESTÁCEAS}

Pesquisas ecológicas indicam que diversas espécies de amebas testáceas são sensíveis às variações ambientais e climáticas e, portanto, podem ser utilizadas como bioindicadores de condições ambientais, tais como temperatura, gradientes das concentrações de oxigênio dissolvido e metais na água (SOUZA, 2008). Dessa forma, estudos realizados com amebas testáceas podem ser importantes ferramentas para o diagnóstico e o planejamento ambiental de ambientes aquáticos, em especial, planícies de inundação brasileiras.

Diversos estudos demonstram o potencial bioindicador desses protistas em respostas à alteração de diversas variáveis ambientais. Um recente estudo realizado por Mieczan et al. (2015) mostrou que determinadas espécies de amebas testáceas apresentam um aumento na abundância de organismos em maiores concentrações de nutrientes, atuando como espécies indicadoras de ambientes produtivos. Ainda nesse contexto, as amebas testáceas são consideradas importantes bioindicadoras do gradiente de temperatura e das mudanças hidrológicas em ambientes de turfeiras (JASSEY et al., 2011; SONG et al., 2014). Além disso, estudos indicam a importância ecológica desses organismos no ciclo biogeoquímico da sílica, e potencialmente, do nitrogênio (AOKI et al., 2007).

Com isso, conhecer a biodiversidade desses organismos representa uma oportunidade de implementar ações de recuperação e conservação de ambientes 
degradados, bem como subsidiar a identificação de áreas prioritárias à preservação e uso sustentável em ambientes aquáticos (RODRIGUES; BICUDO; MOSCHINICARLOS, 2003), principalmente em ambientes de planície de inundação neotropicais.

\section{CONSIDERAÇÕES FINAIS}

Estudos desenvolvidos com amebas testáceas representam a compreensão da dinâmica e estrutura da comunidade e, consequentemente, o funcionamento dos ecossistemas. Essa abordagem pode ser destacada em ambientes de planície de inundação devido à sua elevada biodiversidade de espécies.

Nesse sentido, sugere-se o desenvolvimento de mais pesquisas realizadas com amebas testáceas como subsídio às ações de restauração e recuperação de ambientes degradados, planejamento e manejo de áreas prioritárias à preservação, bem como uso sustentável dos ecossistemas, especialmente em planícies de inundação.

\section{AGRADECIMENTOS}

Agradecemos ao Dr. Moacyr Serafim Júnior pelas sugestões e contribuições a esse trabalho. Os autores também agradecem ao Conselho Nacional de Desenvolvimento Científico e Tecnológico (PELD-CNPQ), Coordenação de Aperfeiçoamento de Pessoal de Nível Superior (CAPES), Fundação Araucária e Núcleo de Pesquisas em Limnologia, Ictiologia e Aquicultura - Universidade Estadual de Maringá (Nupélia/UEM) pelo apoio logístico e financeiro.

\section{REFERÊNCIAS}

AGOSTINHO, A. A.; VAZOLLER, A. E. A. M.; THOMAZ, S. M. The high Paraná river basin: limnological and ichthyological aspects. In: TUNDISI, J. G.; BICUDO, C. M.; MATSUMURA-TUNDISI, T. Limnology in Brazil. Rio de Janeiro: ABC/SBL, 1995. p. 59-104. 
AGOSTINHO, A. A.; ZALEWSKI, M. A planície alagável do alto rio Paraná: importância e preservação. (Upper Paraná River Floodplain: Importance and Preservation). Maringá: Eduem, 1996.

AGOSTINHO, A. A.; THOMAZ, S. M.; NAKATANI, K. A planície de inundação do alto rio Paraná: aspectos físicos, biológicos e socioeconômicos. Maringá: Eduem, 2001.

AGOSTINHO, A. A.; THOMAZ, S. M.; GOMES, L. C. Threats for biodiversity in the floodplain of the Upper Paraná River: effects of hydrological regulation by dams. Ecohydrology and Hydrobiology, v. 4, p. 255-268, 2004.

AOKI, Y.; HOSHINO, M.; MATSUBARA, T. Silica and testate amebae in a soil under pine-oak forest. Geoderma, v. 142, p. 29-35, 2007.

ALVES, G. M.; LANSAC-TÔHA, F. A.; VELHO, L. F. M.; JOKO, C. Y.; COSTA, D. M. New records of testate amoebae (Protozoa: Testacealobosea) for upper Paraná river floodplain. Acta Limnologica Brasiliensia, v. 19, p. 175-195, 2007.

ALVES, G. M.; LANSAC-TÔHA, F. A.; TAKAHASHI, E. M.; VELHO, L. F. M. Fluctuations of testate amoebae populations (Rhizopoda) in plankton from different environments on the upper Paraná river floodplain, Brazil. International Review of Hydrobiology, v. 93, p. 227-242, 2008.

ALVES, G. M.; VELHO, L. F. M.; SIMÕES, N. R.; LANSAC-TÔHA, F. A. Biodiversity of testate amoebae (Arcellinida and Euglyphida) in different habitats of a lake in the upper Paraná floodplain. European Journal of Protistology, v. 46, p. 310-318, 2010.

ALVES, G. M.; VELHO, L. F. M.; COSTA, D. M.; LANSAC-TÔHA, F. A. Size structure of testate amoebae (Arcellinida and Euglyphida) in different habitats from a lake in the upper Paraná River floodplain. European Journal of Protistology, v. 48, p. 169$177,2012$.

ARRIEIRA, R. L.; ALVES, G. M.; SCHWIND, L. T. F.; LANSAC-TÔHA, F. A. Local factors affecting the testate amoeba community (Protozoa: Arcellinida; Euglyphida) in a Neotropical floodplain. Journal of Limnolog y, v. 74, n. 3, p. 444-452, 2015a. 
ARRIEIRA, R. L.; SCHWIND, L. T. F.; BONECKER, C. C.; LANSAC-TÔHA, F. A. Use of functional diversity to assess determinant assembly processes of testate amebae community. Aquatic Ecology, v. 49, p. 561-571, 2015b.

BALIK, V.; SONG, B. Benthic freshwater testate amoebae assemblages (Protozoa: Rhizopoda) from lake Dongting, People's Republic of China, with description of a new species from the genus Collaripyxidia. Acta Protozoologica, v. 39, p. 149-156, 2000.

BEYENS, L.; MEISTERFELD, R. Protozooa: testate amoebae. In: SMOL, J. P.; BIRKS, H. J. B.; LAST, W. B. Tracking Environmental Change Using Lake Sediments. Volume 3: Terrestrial, Algal, and Siliceous Indicators. Dordrecht, The Netherlands: Kluwer Academic Publishers, 2001.

BOBROV, A. A.; CHARMAN, D. J.; WARNER, B. G. Ecology of testate amoebae (Protozoa: Rhizopoda) on peatlands in western Russia with special attention to niche separation in closely related taxa. Protist, v. 150, p. 125-136, 1999.

BONECKER, C. C.; SIMÕES, N. R.; MINTE-VERA, C. V.; LANSAC-TÔHA, F. A.; VELHO, L. F. M.; AGOSTINHO, A. A. Temporal changes in zooplankton species diversity in response to environmental changes in alluvial valley. Limnologica, v. 43, p. 114121, 2013.

CARVALHO, N. O. Hidrologia da Bacia do Alto Paraguai. In: SIMPÓSIO SOBRE RECURSOS NATURAIS E SÓCIO-ECONÔMICOS DO PANTANAL (UFMS, CORUMBÁ, 1984), 1., 1986, Brasília. Anais... Brasília: Embrapa, CPAP, 1986. p. 43-49.

CARVALHO, P.; BINI, L. M.; THOMAZ, S. M.; OLIVEIRA, L. G.; ROBERTSON, B.; TAVECHIO, W. L. G.; DARWISCH, A. J. Comparative limnology of South-American lakes and lagoons. Acta Scientiarum. Biological Sciences, v. 23, p. 265-273, 2001.

CHARMAN, D. J. Biostratigraphic and palaeoenvironmental applications of testate amoebae. Quaternary Science Reviews, v. 20, p. 1753-1764, 2001.

DABÉS, M. B. G. S.; VELHO, L. F. M. Assemblage of testate amoebae (Protozoa, Rhizopoda) associated to aquatic macrophytes stands in a marginal lake of the São 
Francisco river floodplain, Brazil. Acta Scientiarum. Biological Sciences, v. 23, p. 299-304, 2001.

ESTEVES, F. A. Fundamentos de Limnologia. Rio de Janeiro: Interciência, 2011.

FERNÁNDEZ, L. Source-sink dynamics shapes the spatial distribution of soil protists in an arid shrubland of northern Chile. Journal of Arid Environments, v. 113, p. 121-125, 2015.

FERREIRA, C. S.; PIEDADE, M. T. F.; WITTMANN, A. O.; FRANCO, A. C. Plant reproduction in the Central Amazonian floodplains: challenges and adaptations. AoB Plants, v. 2010, p. plq009, 2010.

FOISSNER, W. Soil protozoa: fundamental problems, ecological significance, adaptations in Ciliates and Testateceans, bioindicators and guide to the literature. Progress in Protistology, v. 2, p. 69-212, 1987.

FOURNIER, B.; MALYSHEVA, E.; MAZEI, Y.; MORETTI, M.; MITCHELL, E. A. D. Toward the use of testate amoeba functional traits as indicator of floodplain restoration success. European Journal of Soil Biology, v. 49, p. 85-91, 2012.

GILBERT, D.; AMBLARDB, C.; BOURDIERB, G.; FRANCEZ, A. J.; MITCHELL, A. D. Le régime alimentaire des Thecamoebiens. L'année Biologique, v. 39, p. 1-12, 2000.

GOPAL, B. The role of ecotones (transition zones) in the conservation and management of tropical inland waters. Mitteilungen Internationale Vereinigung für theoretische und angewandte Limnologie, v. 24, p. 17-25, 1994.

HARDOIM, E. L.; HECKMAN, C. W. The seasonal succession of biotic communities in wetlands of the tropical wet and dry climatic zone: IV. Free living sarcodines and ciliates of the Pantanal of Mato Grosso, Brazil. Internationale Revue der gesamten Hydrobiologie und Hydrographie Journal, v. 81, p. 367-384, 1996.

HARDOIM, E. L. Taxonomia e ecologia de Testacea (Protista, Rhizopoda) do Pantanal do Poconé, Rio Bento Gomes e vazante Birici, Mato Grosso, Brasil. 1997. 343f. Tese (Doutorado) - Universidade Federal de São Carlos, São Carlos. 
JASSEY, V.; GILBERT, D.; BINET, P.; TOUSSAINT, M. L.; CHIAPUSIO, G. Effect of a temperature gradient on Sphagnum fallax and its associated living microbial communities: a study under controlled conditions. Canadian Journal of Microbiology, v. 57, p. 226-235, 2011.

JUNK, W. J.; BAILEY, P. B.; SPARKS, R. E. The flood pulse concept in river-floodplain systems. Canadian Journal of Fisheries and Aquatic Sciences, v. 106, p. 110-127, 1989.

JUNK, W. J. Wetlands of Tropical South America. In: WHIGHAM, D.; HEJNY, S.; DYKYJOVA, D. Wetlands of the world. Boston: Dr. W. Junk Publishes, 1993. p. 679739.

JUNK, W. J. Ecology of floodplain: a challenge for tropical limnology. In: SCHIEMER, F.; BOLAND, K. T. Perspectives in Tropical Limnology. Amsterdam: Academic Publishing, 1996. p. 255-265.

LANSAC-TÔHA, F. A.; VELHO, L. F. M.; ZIMMERMANN-CALLEGARI, M. C.; BONECKER, C. C. On the occurrence of testate amoebae (Protozoa, Rhizopoda) in Brazilian inland waters. I. Family Arcellidae. Acta Scientiarum. Biological Sciences, v. 22, p. 355-363, 2000.

LANSAC-TÔHA, F. A.; VELHO, L. F. M.; BONECKER, C. C. Influência de macrófitas aquáticas sobre a estrutura da comunidade zooplanctônica. In: THOMAZ, S. M.; BINI, L. M. Ecologia e manejo de macrófitas aquáticas. Maringá: Eduem, 2003. p. 231-242.

LANSAC-TÔHA, F.A.; VELHO, L. F. M.; BONECKER, C.C. Composition, species richness and abundance of the zooplankton community. THOMAZ, S. M.; AGOSTINHO, A. A.; HAHN, N. S. The Upper Paraná River and its floodplain: physical aspects, ecology and conservation. Leiden: Backhuys Publishers, Leiden, 2004. p. 145-190.

LANSAC-TÔHA, F. A.; ZIMMERMANN-CALLEGARI, M. C.; ALVES, G. M.; VELHO, L. F. M.; FULONE, L. J. Species richness and geographic distribution of testate amoebae 
(Rhizopoda) in Brazilian freshwater environments. Acta Scientiarum. Biological Sciences, v. 29, p. 185-195, 2007.

LANSAC-TÔHA, F. A.; ALVES, G. M.; VELHO, L. F. M.; ROBERTSON, B. A.; JOKO, C. Y. Composition and occurrence of testate amoebae in the Curuá-Una reservoir (State of Pará, Brazil). Acta Limnologica Brasiliensia, v. 20, p. 177-195, 2008.

LANSAC-TÔHA, F. A.; BONECKER, C. C.; VELHO, L. F. M.; SIMÕES, N. R.; DIAS, J. D.; ALVES, G. M.; TAKAHASHI, E. M. Biodiversity of zooplankton communities in the upper Paraná river floodplain: interannual variation from long-term studies. Brazilian Journal of Biology, v. 69, p. 539-549, 2009.

LATRUBESSE, E.; STEVAUX, J. Geomorphology and Environmental aspects of the Araguaia fluvial basin, Brazil. Zeitschrift für Geomorphologie, v. 129, p. 109-127, 2002.

MANTOVANO, T.; ARRIEIRA, R. L.; SCHWIND, L. T. F.; BONECKER, C. C.; LANSACTÔHA, F. A. Rotifer community structure along a stretch under the influence of dams in the Upper Paraná River floodplain. Acta Scientiarum. Biological Sciences, v. 37, p. 281-289, 2015.

MIECZAN, T.; ADAMCZUK, M.; PAWLIK-SKOWRONSKA, B.; TOPOROWSKA, M. Eutrophication of peatbogs: consequences of $\mathrm{P}$ and $\mathrm{N}$ enrichment for microbial and metazoan communities in mesocosm experiments. Aquatic Microbiology Ecology, v. 74, p. 121-141, 2015.

MITCHELL, E. A. D.; WARNER, B. G.; BUTTLER, A.; GOBAT, J. M. Ecology of testate amoebae (Protozoa: Rhizopoda) in Sphagnum peatlands in the Jura mountains, Switzerland and France. Écoscience, v. 6, p. 565-579, 1999.

MITCHELL, E. A. D.; CHARMAN, D. J.; WARNER, B. G. Testate amoebae analysis in ecological and paleoecological studies of wetlands: past, present and future. Biodiversity and Conservation, v. 17, p. 2115-2137, 2008.

NEIFF, J. J. Ideas para la interpretacion ecológica del Paraná. Caracas: Interciencia, 1990. 
NEIFF, J. J. Large rivers of South America: toward the news approach. Verhandlungen des Internationalen Verein Limnologie, v. 26, p. 167-181, 1996.

NEIFF, J. J. Diversity in some tropical wetland systems of South América. In: GOPAL, B.; JUNK, W. J.; DAVIS, J. A. Biodiversity in wetlands: assessment, function and conservation. Leiden: Backuys Publishers, 2001. p. 157-186.

PENNAK, R. W. Fresh-water invertebrates of the United States. New York: The Ronald Press Company, 1953.

ROBERTO, M. C.; SANTANA, N. F.; THOMAZ, S. M. Limnology in the Upper Paraná River floodplain: large-scale spatial and temporal patterns, and the influence of reservoirs. Brazilian Journal of Biology, v. 69, p. 717-725, 2009.

ROCHA, R. R. A.; THOMAZ, S. M. Variação temporal de fatores limnológicos em ambientes da planície de inundação do alto rio Paraná (PR/MS-Brasil). Acta Scientiarum. Biological Sciences, v. 26, p. 261-271, 2004.

RODRIGUES, L.; BICUDO, D. C.; MOSCHINI-CARLOS, V. O papel do perifíton em áreas alagáveis e nos diagnósticos ambientais. In: THOMAZ, S. M.; BINI, L. M. Ecologia e manejo de macrófitas aquáticas. Maringá: Eduem, 2003. p. 211-242.

SCHWIND, L. T. F.; DIAS, J. D.; JOKO, C. Y.; BONECKER, C. C.; LANSAC-TÔHA, F. A. Advances in studies on testate amoebae (Arcellinida and Euglyphida): a scientometric approach. Acta Scientiarum. Biological Sciences, v. 35, p. 549-555, 2013.

SCHWIND, L. T. F.; ARRIEIRA, R. L.; DIAS, J. D.; SIMÕES, N. R.; BONECKER, C. C.; LANSAC-TÔHA, F. A. Structure of the planktonic communities of testate amoebae (Arcellinida and Euglyphida) of three environments of the Upper Paraná River basin, Brazil. Journal of Limnology, v. 75, p. 78-89, 2016.

SMITH, H. G.; BOBROV, A.; LARA, E. Diversity and biogeography of testate amoebae. Biodiversity and Conservation, v. 17, p. 329-343, 2008.

SNEGOVAYA, N.; ALEKPEROV, I. New testate amoebae (Protozoa, Testacea) from the Northern-East Azerbaijan inland water bodies. Protistolog y, v. 6, p. 111-125, 2009. 
SONG, L.; LI, H.; WANG, K.; WU, D.; WU, H. Ecology of testate amoebae and their potential use as palaeohydrologic indicators from peatland in Sanjiang Plain, Northeast China. Frontier of Earth Science, v. 8, p. 564-572, 2014.

SOUZA, M. B. G. Tecamebas (Protozoa Rhizopoda) associadas às macrófitas aquáticas da bacia do rio Jequitinhonha: Parque Estadual do Rio Preto e Parque Estadual do Grão Mogol, MG. Unimontes Científica, v. 2, p. 129-142, 2005.

SOUZA, M. B. G. Guia das tecamebas. Bacia do rio Peruaçu - Minas Gerais. Subsídio para conservação e monitoramento da Bacia do Rio São Francisco. Belo Horizonte: Editora UFMG, 2008.

SOUZA-FILHO, E. E.; STEVAUX, J. C. Geology and geomorphology of the BaíaCurutuba-Ivinhema river complex. In: THOMAZ, S. M.; AGOSTINHO, A. A.; HAHN, N. S. The Upper Paraná River and its floodplain: physical aspects, ecology and conservation. Leiden: Backhuys Publishers, 2004. p. 1-29.

STANFORD, J. A.; WARD, J. V. The hyporheic habitat of river ecosystems. Nature, v. 335, p. 64-66, 1988.

THOMAZ, S. M.; ROBERTO, M. C.; BINI, L. M. Caracterização limnológica dos ambientes aquáticos e influência dos níveis fluviométricos. In: VAZOLLER, A. E. A. M.; AGOSTINHO, A. A.; HAHN, N. S. A planície de inundação do alto rio Paraná: aspectos físicos, biológicos e socioeconômicos. Maringá: Eduem, 1997, p. 73-102.

THOMAZ, S. M.; PAGIORO, T. A.; PADIAL, A. A.; CARVALHO, P. Decomposição das macrófitas aquáticas e sua relação com o pulso de inundação. In: HENRY, R. Ecótones nas interfaces dos ecossistemas aquáticos. Botucatu: UNESP, 2003. p. 195-211.

THOMAZ, S. M.; AGOSTINHO, A. A.; HAHN, N. S. The Upper Paraná River and its Floodplain: Physical aspects, ecology and conservation. Leiden: Backhuys Publishers, 2004.

THOMAZ, S. M.; BINI, L. M.; BOZELLI, R. L. Floods increase similarity among aquatic habitats in river-floodplain systems. Hydrobiologia, v. 579, p. 1-13, 2007. 
TOCKNER, K.; MALARD, F.; WARD, J. V. An extension of the flood pulse concept. Hydrological Processes, v. 14, p. 2861-2883, 2000.

TODOROV, M.; GOLEMANSKY, V.; BLAGOVEST, T. Diversity and biotopic distribution of testate amoebae (Protozoa: Arcellinida and Euglyphida) in the Batak reservoir (Southern Bulgaria). Acta Zoologica Bulgarica, v. 60, p. 115-124, 2008.

TORRES, V. S.; JEBRAM, D. H. A. Amebas testáceas ocorrentes na região de Porto Alegre-RS. Biotemas, v. 7, p. 65-78, 1994.

TORRES, V. S.; SCHWARZBOLD, A. Amebas testáceas ocorrentes na região de Porto Alegre-RS. III. Novos registros de Testaceafilosea (Protoctista: Rhizopoda). Comunicações do Museu de Ciências e Tecnologia da PUCRS, v. 13, p. 139-146, 2000.

TUNDISI, J. G.; MATSUMURA-TUNDISI, T. Limnologia. São Paulo: Oficina de Textos, 2008.

TSYGANOV, A. N.; EMBULAEVA, E. A.; MAZEI, Y. Distribution of soil testate amoeba assemblages along catenas in the northern taiga zone (Karelia, Russia). Protistology, v. 7, p. 71-78, 2012.

VELHO, L. F. M.; LANSAC-TÔHA, F. A.; BINI, L. M. Spatial and temporal variation in densities of testate amoebae in the plankton of the upper Paraná river. Hydrobiologia, v. 411, p. 103-113, 1999.

VELHO, L. F. M.; LANSAC-TÔHA, F. A.; BONECKER, C. C.; ZIMMERMANN-CALLEGARI, M. C. On the occurrence of testate amoebae (Protozoa, Rhizopoda) in Brazilian inland waters. II. Families Centropyxidae, Trigonopyxidae and Plagiopyxidae. Acta Scientiarum. Biological Sciences, v. 22, p. 365-374, 2000.

VELHO, L. F. M.; LANSAC-TÔHA, F. A.; BINI, L. M. Influence of environmental heterogeneity on the structure of testate amoebae (Protozoa, Rhizopoda) assemblages in the plankton of the upper Paraná river floodplain, Brazil. International Review in Hydrobiology, v. 88, p. 154-166, 2003. 
VELHO, L. F. M.; BINI, L. M.; LANSAC-TÔHA, F. A. Testate amoebae (Rhizopodea

- Sarcodina) diversity in plankton of the upper Paraná River floodplain, Brazil. Hydrobiologia, v. 523, p. 103-111, 2004.

WARD, J. V.; STANFORD, J. A. Ecological connectivity in alluvial river ecosystems and its disruption by flow regulation. Regulated Rivers Research and Management Journal, v. 11, p. 105-109, 1995.

WARD, J. V.; TOCKNER, K.; ARSCOTT, D. B.; CLARET, C. Riverine landscape diversity. Freshwater Biology, v. 47, p. 517-539, 2002.

Recebido em: 27 de abril de 2015 Aceito em: 21 de fevereiro de 2016 